\title{
Visuelle Rehabilitation bei Aphakie nach kongenitaler Katarakt
}

\author{
E. Moser, A. Müllner-Eidenböck, C. Weinzierl, A. Thaler und G. Dorner
}

Ambulanz für Pädiatrische Ophthalmologie und Strabismus

Klinik für Augenheilkunde und Optometrie, Medizinische Universität Wien (Vorstand: o. Univ.-Prof. Dr. Ursula Schmidt-Erfurth)

Problemstellung: Die visuelle Rehabilitation nach Operation einer kongenitalen ein- oder beidseitigen Katarakt ist eine Herausforderung für den Augenarzt und die Eltern der kleinen Patienten. Die Korrektur der Aphakie bei Kindern unter 18 Monaten durch Kontaktlinse oder Brille und eine gleichzeitig erforderliche Amblyopiebehandlung sollen zu einer möglichst optimalen visuellen Entwicklung führen.

Methode und/oder Patienten: Wir versorgten $62 \mathrm{Kin}-$ der im Alter unter 18 Monaten (29 mit einseitiger und 33 mit beidseitiger Aphakie) mit Kontaktlinsen und im Fall von Kontaktlinsenproblemen oder -unverträglichkeit mit Brille. Es werden die speziellen Probleme und Anforderungen bei der Kontaktlinsenanpassung in diesem Alter beschrieben. Weiters wird auf die Amblyopiebehandlung, die verschiedenen Formen des bei den aphaken Kindern aufgetretenen Strabismus und dessen Therapie eingegangen.

Ergebnisse: Eine optische Korrektur der Aphakie war in fast allen Fällen mit Kontaktlinse oder Brille gut möglich.
Die visuellen Ergebnisse waren bei beidseitiger Aphakie deutlich besser als bei einseitiger, zum größten Teil abhängig vom Zeitpunkt der Kataraktoperation und der Compliance bei Kontaktlinsen - oder Brillenkorrektur und der Amblyopiebehandlung. Die erforderlichen Schieloperationen waren in fast allen Fällen erfolgreich, ein Stereosehen konnte aber in keinem Fall erreicht werden.

Schlussfolgerungen: Die visuelle Rehabilitation bei Kindern unter 18 Monaten, nach Operation einer kongenitalen Katarakt mittels Kontaktlinse oder Brille, ist noch immer gut möglich, auch wenn in Zukunft Kontaktlinse und Brille immer mehr durch Linsen-Implantation auch in diesem Alter ersetzt werden.

$\mathrm{Zu}$ einem guten Gelingen trägt nicht nur die optische Korrektur, sondern auch die Amblyopiebehandlung und eine eventuell erforderliche Schieloperation bei.

Schlüsselwörter: Aphakie nach kongenitaler Katarakt, Kontaktlinsenanpassung, Amblyopiebehandlung.

\section{Bilaterale Katarakt als Teilsymptom einer phäno- und genotypisch neu identifizierten Multisystem-Krankheit: Das „Congenital Cataracts Facial Dysmorphism Neuropathy (CCFDN)“-Syndrom}

\section{A. Müllner-Eidenböck, E. Moser und M. Amon}

Ambulanz für Pädiatrische Ophthalmologie und Strabismus, Klinik für Augenheilkunde und Optometrie, Medizinische Universität Wien, Österreich (Vorstand: o. Univ.-Prof. Dr. Ursula Schmidt-Erfurth)

Problemstellung: Eines der Teilziele unserer prospektiven Kinderkatarakt-Studie an der Wiener Augenklinik, die wir seit 1998 durchführen, war die Abklärung und Erforschung ätiologischer Faktoren. Die exakte Phänotypisierung von Syndromen mit Augenbeteiligung, ein interdisziplinäres Arbeitsgebiet der Kinderophthalmologie und Pädiatrie, ist Voraussetzung für die rasche Genotypisierung der noch unbekannten Genmutation eines Syndroms.

Methode und/oder Patienten: Im Rahmen der Analyse der ätiologischen Faktoren, die streng altersbezogen (Gruppe $1=$ Säuglinge $0-1,5 \mathrm{a}$, Gruppe $2=$ Vorschulkinder $1,6-4,9 \mathrm{a}$ und Gruppe 3 = Schulkinder/Jugendliche 6-16 a) durchgeführt wurde, identifizierten wir eine Gruppe von 4 Säuglingen mit charakteristischen perioperativen Augenproblemen. Während der ersten Lebensjahre war eine progrediente periphere Neuropathie und die Entwicklung von dysmorphischen Gesichtszügen auffällig. Aus unseren retrospektiven Daten konnten wir noch 5 ältere Kinder mit einem ähnlichen klinischen Bild zuordnen. Aufgrund des klinischen Verlaufes ergab sich der Verdacht auf das Vorliegen des autosomal rezessiven CCFDN-Syndroms, das erst kürzlich (1999) von Tournev et al. bei einer endogamen Gruppe der Vlax Roma in Bulgarien beschrieben wurde. Der Genlocus wurde kurz darauf von Kalaydjieva et al. (Perth/Australien) am Chromosom 18qter lokalisiert.

Unsere 9 Patienten wurden in Zusammenarbeit mit dieser internationalen Forschungsgruppe, die sich auf Erbkrankheiten bei der Roma-Bevölkerung spezialisiert hat, phäno- und genotypisiert.
Ergebnisse: Die 9 Patienten stammten aus 5 miteinander mehrfach verwandten Familien, die alle in der letzten Generation aus einer bestimmten Region in Serbien (Kucevo) nach Wien siedelten. Alle Patienten zeigten die klinischen Kardinalsymptome der Erkrankung (beidseitige kongenitale Katarakt, Mikrocorneae, periphere hypomyelinisierende Neuropathie, milde mentale Retardierung und Gesichtsdysmorphie). Die genetische Analyse ergab, dass alle Index-Patienten homozygot in der CCFDN-Region am Chromosom 18qter waren, während nahezu alle Angehörigen als heterozygote Anlageträger identifiziert werden konnten.

Aufgrund unseres Kinder-Katarakt-Studienprotokolles konnten wir daher die detaillierte okuläre Symptomatik des Syndroms bei einer genetisch identifizierten Patientengruppe beschreiben (Ophthalmology 2004; 111: 1415-1423). Im September 2003 erfolgte dann die CCFDN-Gen-Identifikation (Nature Genet 2003; 35: 185-189). Die Transition einer einzigen Base (C->T) in einer sehr frühen Phase der Transkription führt zum partiellen Defizit der C-terminalen Domäne Phosphatase, einer essentiellen Komponente der eukaryoten Transkriptions-Maschinerie. Demnach gehört das CCFDN-Syndrom zu den sehr seltenen Transkriptions-Syndromen.

Schlussfolgerungen: Die praktische Relevanz einer zielstrebigen Abklärung der Katarakt-Ursache bei Säuglingen ist groß: von klein auf können diese Kinder interdisziplinär (neurologisch, physiotherapeutisch, ophthalmologisch, orthopädisch, intellektuell) betreut und gefördert werden. Damit können Sekundär-Erkrankungen wie hochgradige 